\title{
Two measurement modes for mobile phone optical sensing
}

\author{
Mats Eriksson and Zafar Iqbal
}

\section{Linköping University Post Print}

\section{Tweet}

N.B.: When citing this work, cite the original article.

Original Publication:

Mats Eriksson and Zafar Iqbal, Two measurement modes for mobile phone optical sensing, 2014, Sensors and actuators. B, Chemical, (195), 63-70.

http://dx.doi.org/10.1016/j.snb.2014.01.005

Copyright: Elsevier

http://www.elsevier.com/

Postprint available at: Linköping University Electronic Press

http://urn.kb.se/resolve?urn=urn:nbn:se:liu:diva-105893 


\title{
Two measurement modes for mobile phone optical sensing
}

\author{
Mats Eriksson, Zafar Iqbal \\ Department of Physics, Chemistry and Biology, Linköping University, SE-581 83 Linköping, Sweden
}

\begin{abstract}
The use of a mobile phone for classification and quantification of liquid samples is described. The screen of the phone is used for controlled illumination and the front side camera for imaging. No additional optical equipment is needed. It is shown that there are different regions of the captured image containing different information about the sample. One region contains light that has been specular reflected at the air-liquid interface and one is dominated by light that has propagated through the liquid. The specular reflected light contains information about the refractive index of the liquid sample whereas the transmitted light contains information about color and absorption. It is found that the specular reflectance increases linearly with increasing refractive index, $n$, in the range $1.33<n<1.38$, as expected from the Fresnel equations. A change of the refractive index of about 0.004 refractive units can be detected. The transmitted light intensity is well described by the Beer-Lambert law over a large absorption range. Light from the two different areas of the image is used to analyze several types of liquid samples. It is shown that a combination of the two measurement modes improves the classification abilities of the mobile phone.
\end{abstract}

Keywords: Mobile phone; optical sensor; refractive index; liquids; absorption

\section{Introduction}

The incorporation of sensors into mobile phones has provided potential for establishing inexpensive mobile sensor systems. Mobile phones are attractive consumer electronic products for mobile sensing, e.g. for home testing, because of their wide availability and high level of familiarity with the general public. The range of sensors available is steadily growing and today includes motion sensors (e.g. accelerometers, gyroscopes and gravity sensors), environmental sensors (e.g. ambient air temperature, pressure and humidity sensors) and position sensors (e.g. GPS and magnetometers) apart from the more obvious microphone, camera and touchscreen $[1,2]$.

The camera in combination with the screen may be used as an optical sensor system. So far, it is mainly the digital camera in the phone that has been utilized for analyses [3-7], but there is also a possibility to take advantage of the phone's screen for versatile sample illumination. The combination of these two features has been demonstrated to enable simple analyses $[8,9]$, which can be considerably improved quantitatively by high dynamic range (HDR) imaging [10] and extended to more advanced measurements by optical add-ons to the mobile phone $[11,12]$. 
The optical properties of liquids are often used for characterization of samples. Transmittance and reflectance measurements give information about the absorption coefficient and the refractive index. Turbidity can be measured by light scattering with a nephelometer. The traditional instrument for refractive index measurements is the Abbe refractometer, but several alternative methods have also been proposed, see e.g. $[13,14]$ and references therein. A multifunctional spectrophotometer capable of measuring optical properties of both transparent and turbid liquid samples has been reported [15]. The technique is based on controlled guidance of the light beam into the sample from desired directions which allows coordinated observation of transmission, reflection and light scattering from samples. The instrumentation is effective for analysis of colorless solutions but has also been used for transparent, absorbing liquid samples. Control of illumination and detection is a considerably more primitive level for the mobile phone optical sensor, as compared to the multifunctional spectrophotometer. However, a simple mobile phone optical sensor would be intended for less demanding applications, like home testing and simple experiments in education, particularly in the developing countries where dedicated laboratory instruments are scarce.

In this communication we show that two different modes of mobile phone optical sensing are available in one and the same image. The mobile phone is kept horizontal during the measurements, i.e. the screen and the camera are parallel to the liquid sample surface. The fixed geometry of the front side camera relative to the screen gives rise to an image of the sample containing an area where specular reflected light from the air-liquid interface contributes to the detected light intensity. In addition, light that is diffuse reflected by the measurement vessel, containing the liquid, contributes to the intensity in the whole image. In a recent publication we showed that this light, which is transmitted through the liquid, contains information about the wavelength dependence of the absorption by colored transparent liquids [16]. For a large absorption range the concentration dependence is well described by the Beer-Lambert law allowing quantification of concentrations. The intensity of the specular reflected light is, on the other hand, determined by the refractive index of the liquid which allows measurements also on colorless solutions. This new possibility to measure refractive index changes of liquids is first investigated and this measurement mode is then used together with the transmission mode to increase the classification power of a mobile phone optical sensor system. Examples are given where the combination of refractive index and absorption measurements are required for the discrimination of the different samples.

\section{Experimental details}

Colorless salt solutions were prepared by dissolving $100 \mathrm{~g}$ sodium chloride in $300 \mathrm{~g}$ deionized water to obtain a 25 weight percent (wt \%) concentration. Dilutions were made with water down to $5 \mathrm{wt} \%$ concentration. Solutions of sucrose, glucose and fructose were prepared in a corresponding manner. Ethanol and methanol were mixed with water to obtain different concentrations in volume percent (vol \%). 
The measurement vessel for the samples was a weighing boat of white, semitransparent polystyrene with dimensions $80 \mathrm{~mm}$ x $80 \mathrm{~mm}$ x $25 \mathrm{~mm}$. A black laminated paper was positioned under the vessel and the phone was placed on a mechanical stand which allowed positioning to establish a distance of $70 \mathrm{~mm}$ between the sample surface and the screen/camera as shown in Fig. 1a. At this distance a suitable field of view was obtained for the fix focus and fix focal length lens of the camera. A black, opaque plastic box was placed over the setup to exclude ambient light during measurements. All measurements were done on $70 \mathrm{ml}$ of sample.

A Nokia 6220 classic mobile phone was used both as light source and detector in the study. The TFT display of the phone served as a controlled illumination source and the front view (secondary) camera recorded images. This camera is designed for video calls and has $176 \times 144$ pixels (QCIF) for video recording with 8 bit resolution in the red (r), green (g) and blue (b) channels. Illuminations were performed with the three primary colors red $(\mathrm{R})$, green (G) and blue (B) and recorded in the red, green and blue channels of the camera, respectively. The spectral properties of the screen and the camera have been described previously [16]. The open source integrated design environment NetBeans was used to design and implement software in the form of a MIDLet (Mobile Information Device applet) which controlled the display as light source and simultaneously acquired images to obtain reflectance intensities from the samples. MATLAB-R2007b and R2010a (MathWorks, Natick, MA, USA) were used for image processing.

As indicated in Fig. 1a, specular reflection (i.e. a mirror image) is expected from part of the cameras field of view. This is confirmed by the captured image in Fig. $1 \mathrm{~b}$ as a high intensity rectangle in the lower part (containing Area 2). Area 2 was used for evaluation of refractive index differences between different liquid solutions. The rest of the image contains no specular reflection and Area 1 was chosen for evaluation of the absorption properties. Area 3 is used for reference purposes.

Area 1 contained $60 \times 100$ pixels, while Areas 2 and 3 contained $40 \times 40$ pixels. The mean value of all pixel data from the area in question and 50 consecutive images from the video was used for data presentation. This means that a data point from Area 1 (Area 2) is the mean value of 300000 (80 000) pixel values. More information about the video recording and the image processing can be found in [16].

\section{Results and discussion}

\subsection{The transmission measurement mode}

Data from Area 1 is (mainly) due to transmission of light from the screen through the liquid followed by diffuse reflection at the liquid-vessel interface and (again) transmission through the liquid. Although this is not an optimal transmission measurement, data from this area resemble those of standard transmission measurements through a liquid as long as scattering contributions are low and is very sensitive to absorption. Since the absorption by colored solutions is wavelength dependent, the data from Area 1 of colored liquid solutions 
can be used to both classify sample identities and quantify sample concentrations [16]. This area may also be influenced by scattering of light and fluorescence in the liquid.

The data of Area 1 of the image is well described by the Beer-Lambert law, i.e. the measured light intensity falls exponentially with increasing concentration [16]. Fig. 1c shows 3D RGB scatter plots for different concentrations of three different colorants added to deionized water. The data are results after a logarithmic linearization, which explains the linear dependence in the figure (the concentration increase is the same between adjacent data points). In this example the RGB measurement thus enables both classification (direction in RGB space) and quantification (distance from the origin). More details about this measurement mode are available in [16].

\subsection{The specular reflection measurement mode}

Area 2 in Fig. $1 \mathrm{~b}$ is strongly influenced by specular reflection of light at the air-liquid interface. This can be utilized to distinguish also non-colored liquid solutions if the samples have different refractive indices. The specular reflectance is dependent on the incidence angle, the polarization of the light and the refractive index of the liquid as illustrated in Fig. 2a and b. The incidence angles relevant for the geometries of this work (see Fig. 1a) are below $15^{\circ}$. In this range the reflectance does not deviate very much from the reflectance at zero incidence angle (normal incidence) as indicated in Fig. 2b. The polarization of the light from the screen was found to be dominated by the perpendicular component for the angles detected by the camera, but it was not purely linearly polarized so there were also contributions from the parallel component. This tends to neutralize the influence of the angular dependence in the studied range. Fig. 2c shows the difference data between two images of 0 and $25 \%$ sodium chloride solutions. An increased light intensity in the high intensity area of Fig. 1b is observed as expected from the refractive index increase. This indicates that refractive index changes can be considered as a fourth dimension of sensing, in addition to the three dimensions illustrated in Fig. 1c.

The reflectance, $R$, at zero incidence angle is, according to the Fresnel equations, given by:

$$
R=\frac{I_{r}}{I_{0}}=\left(\frac{n_{i}-n_{t}}{n_{i}+n_{t}}\right)^{2}
$$

where $I_{0}$ is the incident light intensity, $I_{r}$ is the reflected intensity, $n_{i}$ is in this case the refractive index of air $\left(n_{i}=1.000\right)$ and $n_{t}$ the refractive index of the liquid. $R$ is thus in general a non-linear function of $n_{t}$ for a constant $n_{i}$, but in the limited region of interest of this work, $1.33<n_{t}<1.38$, the reflectance is to a good approximation linearly dependent on $n_{t}$ as illustrated in Fig. 3a.

The intensity of light in Area 2 was measured for different concentrations of sodium chloride. Fig. $3 b$ shows the results for measurements with green illumination from the screen measured in the green channel of the image sensor ("Experimental, raw data"). A linear 
increase with increasing salt concentration is observed and the detected intensity for $25 \mathrm{wt} \%$ salt concentration is about $4 \%$ higher than for $0 \%$ salt.

The expected increase in reflectance as a function of salt concentration has also been calculated. The refractive indices at $589 \mathrm{~nm}$ for $0,5,10,15,20$ and $25 \mathrm{wt} \%$ sodium chloride in water are 1.3334, 1.3418, 1.3488, 1.3594, 1.3684 and 1.3776, respectively [17]. Applying eq. (1) yields the "Theoretical" graph in Fig. 3b. Thus, we expect an increase of the reflectance with about $23 \%$, i.e. about 6 times higher than observed in the experimental data.

A large part of this discrepancy is due to the fact that the light that reaches the camera from Area 2 is not only specular reflected light. A large fraction of the light is actually diffuse reflected light from the liquid-vessel interface. If we approximate this part of the total light intensity with the intensity measured in Area 3 of Fig. 1b, we can estimate the specular reflected intensity by taking the difference between the intensities measured in Area 2 and Area 3 of Fig.1b. This procedure raises the experimental graph by about a factor of three. There are, however, still several non-ideal conditions in the measurements. The illumination is not uniform across the image, the angle of incidence varies and we conduct the measurements with a partially polarized light. Furthermore, the camera utilizes an automatic exposure that might influence the results. We consider the non-uniform illumination to be the main reason for the remaining discrepancy. In order to investigate the importance of this factor, a dry measurement vessel was imaged and it was found that the illumination was $15 \%$ brighter in Area 2 than in Area 3. By correcting also for this fact, the "Experimental, adjusted" graph of Fig. 3b is obtained, which accounts for most of the discrepancy between the "Theoretical" and the "Experimantal, raw data" graphs. (Note, however, that the adjusted data point for $5 \%$ salt concentration coincides with the corresponding "Experimental, raw data" data point).

A good understanding of the main processes giving rise to the experimental raw data graph has thus been obtained. It is, however, not crucial to know all the details. For sensing purposes it is more important that the experimental raw data graph is linear (as expected) and shows a very small standard deviation. This means that only a simple calibration is needed to convert the experimental raw data to refractive index, if absolute values are required. The "Experimental, raw data" was therefore used in the remainder of the data evaluations below.

In Fig. 4 data from Area 2 (of Fig. 1b) is compared with data from Area 1. In both cases green illumination $(\mathrm{G})$ is used and the light is detected in the green channel of the image sensor $(\mathrm{g})$. This combination is called $\mathrm{Gg}$ in the figure. The data from Area 2 is called $\mathrm{sGg}$ (with $\mathrm{s}$ as in specular reflection) and the data from Area 1 is called $\mathrm{tGg}$ (with $\mathrm{t}$ as in transmission). The sGg data is the same as the "Experimental, raw data" of Fig. 3b and the relative reflectance increases with about $4 \%$ when the salt concentration increases from 0 to $25 \mathrm{wt} \%$. Note though that a different scale is used which enables a study of the standard deviation of the data of Fig. 4. The tGg data indicates a decrease with increasing salt concentration, even if the change is both small and the data noisy on this scale. As illustrated in Fig. 2b, when the refractive index increases from 1.33 (0\% salt) to 1.38 (25\% salt) the reflectance (at zero incidence angle) is expected to increase from 0.020 to about 0.025 . i.e. 
with about $25 \%$. If there is no absorption in the liquid the corresponding transmissions of light into the water should therefore decrease from 0.980 to 0.975 , i.e. a decrease of only $0.5 \%$. The same loss of intensity occurs again when light that is diffusively reflected at the bottom of the vessel is transmitted from the liquid back into air. In total, a decrease of the intensity of the diffusively reflected light is expected to be about $1 \%$. Thus, if there is a change in the refractive index of the liquid, it is expected to influence both the specular reflected light (contributing to the $\mathrm{sGg}$ mode) and the light transmitted into the water (contributing to the $\mathrm{tGg}$ mode). The effect is expected to be much larger for the sGg mode in agreement with the experimental data of Fig. 4.

Several different substances forming non-colored transparent solutions with water were investigated and the results for three of them are shown in Fig. 5a. As expected, substances that give large changes of the refractive index compared to water (sodium chloride and sucrose in Fig. 5a) give rise to a large slope in the concentration dependence, where all different concentrations can be distinguished, while substances that give only a small change (ethanol) give a small slope. The refractive indices of the studied substances at $25 \%$ (wt \% for sodium chloride and sucrose and vol \% for ethanol) are 1.378 for sodium chloride, 1.372 for sucrose and 1.347 for ethanol for light with a wavelength of $589 \mathrm{~nm}$ [17]. These values should be compared with that of water, 1.333. Solutions of glucose and fructose were also studied and gave similar results as sucrose. This is expected since they have very similar refractive indices [17]. Methanol solutions were also studied, but since the refractive index is close to that of water (1.338 for a $25 \%$ methanol solution), quantification of these samples was not possible.

In Fig. 5b the same data as in Fig. 5a is plotted as a function of refractive index. The concentration data has been converted to refractive index from experimental data in [17]. It should be emphasized that the reference refractive index data was measured at a well-defined wavelength, the sodium D line at $589 \mathrm{~nm}$, while the mobile phone data was measured with a much broader spectrum of 480-590 $\mathrm{nm}$ [16]. The uncertainty in the refractive index is therefore rather large for the different substances. Nevertheless, the data clearly indicates a linear dependence of the relative reflectance on the refractive index. A detection limit of a 0.004 refractive units change can be estimated based on three times the standard deviation of the data at zero concentration. This is of course not comparable to dedicated instruments but might be useful in less demanding applications.

\subsection{Applications of the two measurement modes}

In a separate paper we have presented how measurements in Area 1 (the $\mathrm{t}$ mode, transmitted light) can be used to classify identities of samples with different colors and to quantify concentrations [16]. We now look at a few applications where both the t mode (sensitive to wavelength dependent absorption) and the s mode (Area 2, specular reflected light, sensitive to changes of the refractive index) are used. Some of the examples show that utilizing a combination of the two measurement modes permits an analysis that would be very difficult if only one of the modes was utilized. 


\subsubsection{Classification of edible oils and fatty acids}

Fig. 6a shows results for measurements on edible oils and pure fatty acids. There are some similarities in the composition of these substances. The major components of both canola oil (rapeseed oil) and sunflower oil are oleic and linoleic fatty acids. For canola oil oleic acid dominates while linoleic acid is normally the major component of sunflower oil [18]. Oleic acid is a cis-fatty acid [19] and nonanoic acid (pelargonic acid) is a saturated fatty acid. The most distinctive visual characteristic of the samples was their yellowish color. Oleic acid exhibited the visually strongest color and this was confirmed by it having the highest absorbance at $470 \mathrm{~nm}$ as determined in a UV-Vis spectrometer, while canola oil and nonanoic acid had the lowest absorbances at $470 \mathrm{~nm}$.

Classification of the oils was possible by combining blue illumination detected in the blue channel in Area 1 of the image (tBb axis in Fig. 6a) with red illumination detected in the red channel in Area 2 (sRr axis). The discrimination along the $\mathrm{tBb}$ axis correlates with the differences in absorbance at $470 \mathrm{~nm}$. The discrimination along the $\mathrm{sRr}$ axis correlates with the differences in refractive index which are 1.434 for nonanoic acid [20], 1.458 for oleic acid [20], 1.466 for canola oil [21] and 1.474 for sunflower oil [21] at $589 \mathrm{~nm}$. The reason for choosing sRr rather than $\mathrm{sGg}$ was that there was a strong influence of absorbance also for the Gg illumination/detection channel combination, which disturbed the sGg measurement. The absorbance was much smaller for the Rr combination.

From Fig. 6a it is clear that for a measurement of only tBb (absorption) it would be very difficult to separate nonanoic acid, canola oil and sunflower oil. Similarly, for a measurement of only sRr (refractive index) it would be difficult to separate oleic acid and canola oil. Only by combining both measurement modes a clear classification of all four substances is possible.

The influence of mixing oil with water was also studied. Different volume percent of de-ionized water was added to the oil and the mixture was then shaken which generated an emulsion. The reflectance in Area 1 for red illumination and red camera channel (Rr value) for the Canola oil is presented in Fig. 6b. A monotonically increasing function of the water content is observed. Since an emulsion was obtained, a large fraction of backscattering of the light occurred which differed from the other solutions studied in this work. The results thus indicate that the technique may have some application also for samples with large light scattering. For the other oils and other measurement combinations the change with water content was not monotonic, but in several cases there was a clear difference between oil with and without water, respectively.

\subsubsection{Glucose concentration measurements}

Figure 7 describes analysis of glucose in water. Fig. 7a shows results for direct analysis of high concentrations by specular reflection from liquid samples. The results are similar to those of sucrose in Fig. 5 and are correspondingly due to the change in the refractive index 
that occurs upon addition of glucose. The sGg measurement combination was applied also in this case in order to capture these changes in refractive index. The standard deviation of the lower concentrations is rather high, hampering accurate measurements in this area. This could probably be improved by more repetitions of these measurements ( 5 repetitions were made).

Test strips (Merck) were used to determine lower glucose concentrations $(50 \mathrm{mg} / \mathrm{l}=50$ $\mathrm{ppm}=0.005 \mathrm{wt} \%$ ) and the results are presented in Fig. 7b. In this measurement approach the phone served as a reader for evaluating the color change resulting from enzymatic action of glucose in the test area. This was a diffuse reflection Gg measurement directly of the test strips and a detection limit less than $20 \mathrm{mg} / \mathrm{l}$ can be estimated. The sGg mode is thus suitable for high glucose concentrations, while the diffuse reflection mode is suitable for low concentrations by combining imaging with commercial test strips. This dual measurement strategy therefore provides a wide concentration range for glucose measurements. The high standard deviation at the lowest concentrations in Fig. $7 \mathrm{~b}$ is, again, probably influenced by the low number of repetitions ( 3 times) of the measurement. The sensitivity and resolution of test strip measurements can be further improved by applying HDR processing [10].

\subsubsection{Ethanol measurements in colored solutions}

A classification of solutions with different ethanol concentrations and different colors is shown in Fig. 8. The blue colorant was reactive blue 2 (VWR, West Chester, PA, USA) and the brown color was obtained by dissolving cane sugar in de-ionized water. All samples of the same color contained the same amount of colorant. The $\mathrm{tBb}$ data separates the different colors due to their different absorption properties and the $\mathrm{sGg}$ data discriminates the different ethanol concentrations due to changes of the refractive index. The sugar in the brown colored solutions gives rise to an increase in the refractive index (see discussion related to Fig. 5) and a slightly higher sGg value is therefore expected, compared to the blue colored solution, for a certain ethanol concentration. This is also what is observed in Fig. 8.

These classifications would be very difficult to carry out if only one of the measurement modes was utilized. If only the $\mathrm{tBb}$ data (absorption) is used, no information about the ethanol concentration can be obtained. If, on the other hand, only the sGg data (refractive index) is used, no information about the liquid color (absorption) is available and since the sugar adds to the refractive index in the brown colored solutions, the sGg value alone is not enough to establish the ethanol concentration. With the combined tBb and sGg data, however, all samples can be classified in a meaningful way.

\section{Conclusions}

The mobile phone is naturally not an optimal device for analytical optical investigations. It is interesting, however, that even in a simple measurement setup and with simple signal evaluation, the use of the different types of information found in the camera image enables new analytical possibilities with the mobile phone. 
The results show that the intensity of the most intense area in the image is correlated with the refractive index of a liquid sample due to specular reflection. In principle it should therefore also be possible to study transparent solids with the technique. Although the dependence of the detected light intensity is not only dependent on the refractive index, also diffuse reflected light contributes, a linear dependence on the refractive index of water solutions is observed, which is in agreement with the Fresnel equations.

In addition to the specular reflection measurement mode, the transmission measurement mode from another part of the image gives information about the wavelength dependent absorption properties, which gives rise to a three dimensional data space (rgb) for classification and quantification of colored solutions [16]. The new specular reflection mode adds a fourth dimension of sensing that allows measurements also on colorless, non-absorbing samples.

The sample volume used in this work may be too large for some applications. The technique outlined here is, however, compatible with much smaller volumes. Also other developments of the technique are possible. The complete measurement, including data evaluation, should for example be integrated in the mobile phone, e.g. as a mobile phone application.

In summary two modes of sensing are available. It is shown that by combining both measurement modes in a single measurement, classification of liquids could be made, where the use of only one of the possibilities would not suffice. We suggest that the possibility demonstrated in the paper can be further developed into a simple and generally available method, e.g. for simple tests of beverages or quality control of cooking oil. Another area of application might be laboratory exercises in education where this versatile and cost effective optical tool already shows potential $[22,23]$.

\section{Acknowledgements}

Z.I. is recipient of a scholarship from Pakistan's Higher Education Commission (HEC). The authors thank Dr. Robert Bjorklund and Prof. Ingemar Lundström for their valuable contributions to the work. 


\section{References}

[1] N. D. Lane, E. Miluzzo, Hong Lu, D. Peebles, T. Choudhury, A.T. Campbell, A survey of mobile phone sensing, Communications Magazine, IEEE , 48 (2010) 140-150.

[2] http://developer.android.com/guide/topics/sensors/sensors_overview.html, accessed May 21, 2013.

[3] A.W. Martinez, S.T. Phillips, E. Carrilho, S.W. Thomas, H. Sindi, G.M. Whitesides, Simple telemedicine for developing regions: camera phones and paper-based microfluidic devices for real-time, off-site diagnosis, Anal. Chem. 80 (2008) 36993707.

[4] B.A. Cadle, K.C. Rasmus, J.A. Varela, L.S. Leverich, C.E. O’Neill, R.K. Bachtell, D.C. Cooper, Cellular phone-based image acquisition and quantitative ratiometric method for detecting cocaine and benzoylecgonine for biological and forensic applications, Subst. Abuse: Res. Treat. 4 (2010) 21-33.

[5] H.P. Ninh, Y. Tanaka, T. Nakamoto, K. Hamada, A bad-smell sensing network using gas detector tubes and mobile phone cameras, Sens. Actuators B 125 (2007) 138-143.

[6] A. García, M.M. Erenas, E. D. Marinetto, C. A. Abad, I. de Orbe-Paya, A. J. Palma, L. F. Capitán-Vallvey, Mobile phone platform as portable chemical analyzer, Sens. Actuators B 156 (2011) 350-359.

[7] H. Zhu, O. Yaglidere, T. Su, D. Tseng, A. Ozcan, Cost-effective and compact widefield fluorescent imaging on a cell-phone, Lab Chip 11 (2011) 315-322.

[8] Z. Iqbal, R.B. Bjorklund, Assessment of a mobile phone for use as a spectroscopic analytical tool for foods and beverages, Int. J. Food Sci. Technol. 46 (2011) 2428-2436.

[9] Z. Iqbal, D. Filippini, Spectral fingerprinting on a standard mobile phone, Journal of Sensors 2010 (2010) Article ID381796, 1-9.

[10] P. Preechaburana, S. Macken, A. Suska, D. Filippini, HDR imaging evaluation of a NTproBNP test with a mobile phone, Biosens. Bioelectron. 26 (2011) 2107-2113.

[11] P. Preechaburana, A. Suska, D. Filippini, Embedded Adaptive Optics for Ubiquitous Lab-on-a-Chip Readout on Intact Cell Phones, Sensors 12 (2012) 8586-8600.

[12] P. Preechaburana, M. C. Gonzalez, A. Suska, D. Filippini, Surface Plasmon Resonance Chemical Sensing on Cell Phones, Angew. Chem. Int. Ed. 51 (2012) 11585 -8.

[13] I. Niskanen, Jukka Räty, R. Myllylä, V. Sutinen, K. Matsuda, K. Homma, P. Silfsten, K-E Peiponen, Roughened glass slides and a spectrophotometer for the detection of the wavelength-dependent refractive index of transparent liquids, Appl. Spec. 66 (2012) 786-790.

[14] C.-C. Hsu. K.-H. Chen, D.-C. Su, Normal incidence refractometer, Optics Communications 218 (2003) 205-211 
[15] I. Niskanen, Jukka Räty, K-E Peiponen, A multifunction spectrophotometer for measurement of optical properties of transparent and turbid liquids, Meas. Sci. Technol. 17 (2006) N87-N91.

[16] Z. Iqbal, M. Eriksson, Classification and quantitative optical analysis of liquid and solid samples using a mobile phone as illumination source and detector, Sens. Actuators B 185 (2013) 354-362.

[17] "Concentrative Properties of Aqueous Solutions: Density, Refractive Index, Freezing Point Depression, and Viscosity" in CRC Handbook of Chemistry and Physics, 92nd Edition (Internet Version 2012), W. M. Haynes, ed., CRC Press/Taylor and Francis, Boca Raton, FL.

[18] F. D. Gunstone, Vegetable Oils, in: Fereidoon Shahidi, (Ed.), Bailey's Industrial Oil and Fat Products, Vol. 1, 6th Edition, John Wiley \& Sons, Inc., Hoboken, NJ, 2005.

[19] N. O. V. Sonntag, Structure and composition of fats and oils, in: Daniel Swern (Ed.), Bailey's Industrial Oil and Fat Products, Vol. 1, 4th Edition, John Wiley \& Sons, Inc., New York, 1979.

[20] "Physical Constants of Organic Compounds" in CRC Handbook of Chemistry and Physics, 93rd Edition (Internet Version 2013), W. M. Haynes, ed., CRC Press/Taylor and Francis, Boca Raton, FL.

[21] "Composition and Properties of Common Oils And Fats" in CRC Handbook of Chemistry and Physics, 93rd Edition (Internet Version 2013), W. M. Haynes, ed., CRC Press/Taylor and Francis, Boca Raton, FL.

[22] A. Scheeline, Teaching, learning, and using spectroscopy with commercial, off-theshelf technology, Applied Spectroscopy. 64 (9) (2010) 256A-268A.

[23] Z. J. Smith, K. Chu, A. R. Espenson, M. Rahimzadeh, A. Gryshuk, M. Molinaro, D. M. Dwyre, S. M. Lane, D. Matthews, S. Wachsmann-Hogiu, Cell-phone-based platform for biomedical device development and education applications, PLoS ONE 6 (2011) e17150 


\section{Biographies}

Mats Eriksson is Associate Professor at Linköping University, Sweden. Eriksson received his $\mathrm{PhD}$ in Applied physics in 1997. He is since 2007 heading a chemical sensor group and was during 2006-2009 working part time as a Senior Scientist at AppliedSensor. His research interest is primarily on chemical sensors applied to liquids and different aspects of gas sensing, in particular with field-effect devices, covering areas such as electrochemistry, microelectrode arrays, surface and interface physics, catalytic reactions, thin film physics, semiconductor physics and optical sensing. He is currently involved in both basic and applied research projects on chemical sensors for drinking water monitoring. Eriksson is also a member of the management board of the scientific research environment Security Link (www.liu.se/security-link) in the technical domain of the Security and Crisis Management area.

Zafar Iqbal received his MSc degree in Physics (major in experimental physics, from the University of Punjab, Lahore, Pakistan) and in Engineering (major in design engineering, from Quaid-e-Azam University, Islamabad, Pakistan) in the years 1988 and 1992, respectively. During 1992-2007 he worked as principal scientist at the department of design engineering at the Pakistan atomic energy commission (PAEC), with mathematical-modeling, software-development and computer-simulations with respect to system design and design evaluation. He has since 2007 been with Linköping University working on different aspects of optical sensing with mobile phones and received his PhD in Applied Physics in 2012. Iqbal is now back at PAEC. 
a)
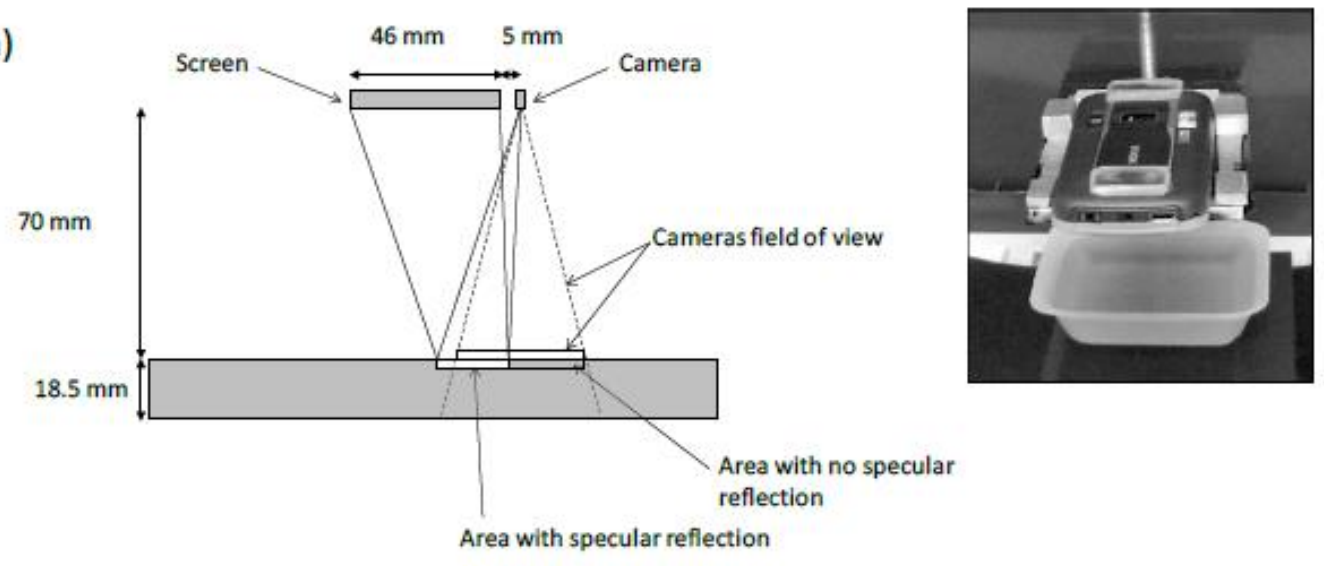

b)

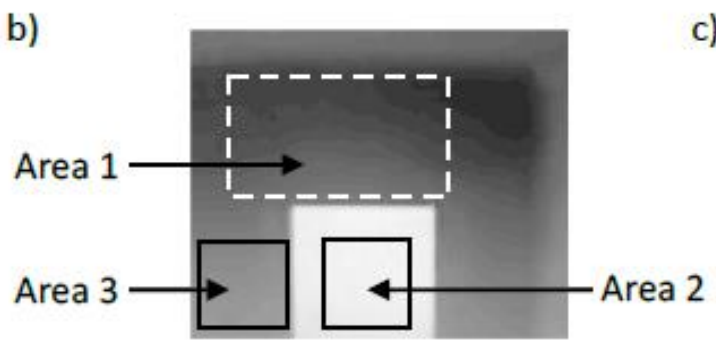

c)

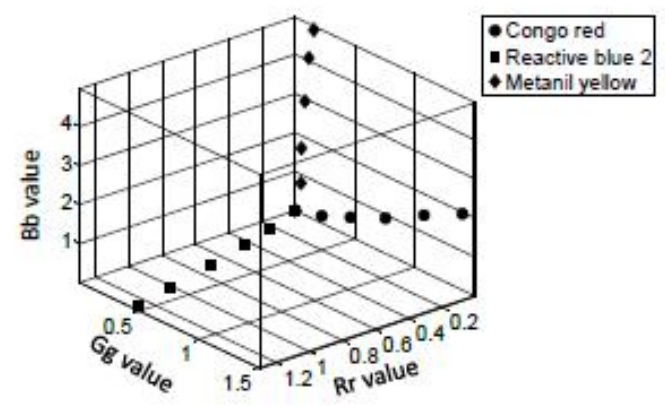

Fig. 1. (a) Experimental set-up schematically viewed from the side (left) and obliquely imaged from above (right). The solid lines indicate the area on the liquid surface that gives rise to specular reflections that reach the position of the camera. The dashed lines indicate the camera's field of view. The "Area with specular reflection" thus reaches the camera, but only part of this area is seen in the image. From Ref. [16]. (b) The average of 50 frames from the video sequence during measurements on de-ionized water. Three different areas for different types of evaluations are marked. The faint outline at the top and to the right of the image is due to the sloping bottom edge of the measurement vessel. (c) An example of results from Area 1, illustrating that this area is suitable for classifying and quantifying colored liquid samples. The capital letter of each axis indicates the illumination color from the screen (red (R), green $(\mathrm{G})$ or blue $(B)$ ), while the lower case letter indicates the channel of the camera ( $r, g$ or b). Results for three different colorants are shown. Different directions in the RrGgBb space are observed for the different colorants and the distance from the origin increases with increasing concentration. From Ref. [16]. 
a)

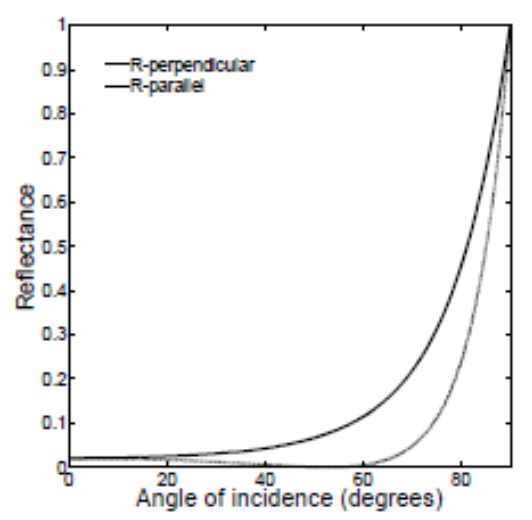

b)

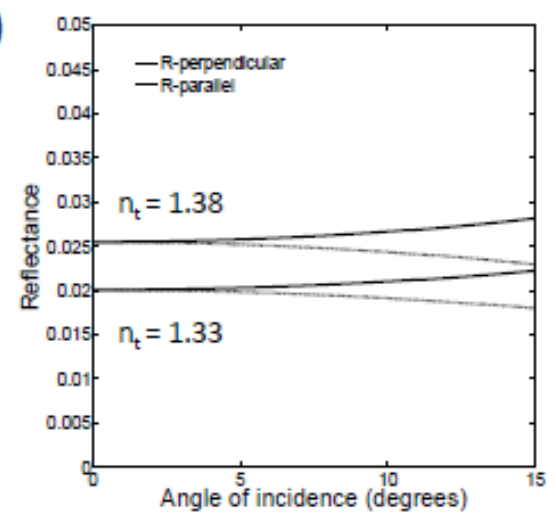

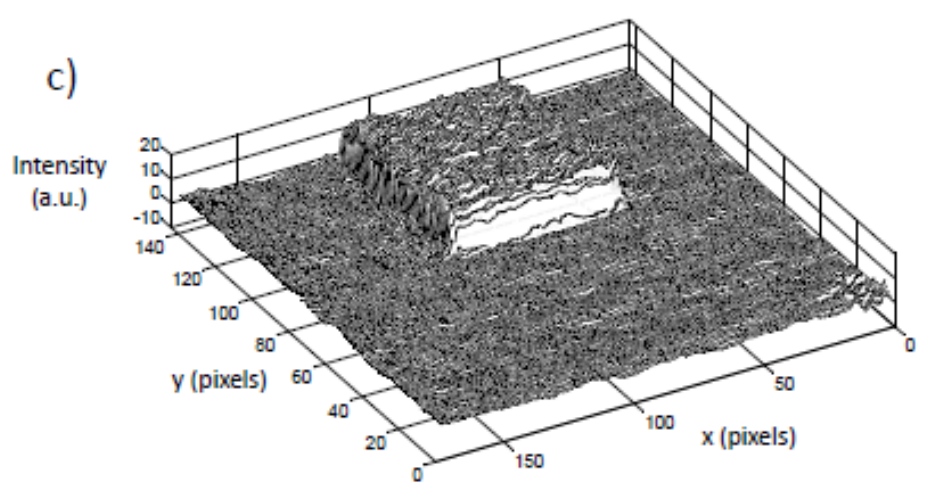

Fig. 2. (a) Specular reflectance as a function of incidence angle for the air-water interface $\left(n_{\text {air }}=\right.$ 1.00 and $\mathrm{n}_{\text {water }}=1.33$ ) according to the Fresnel equations. R-perpendicular (solid line) is the component of the electrical field oscillating in a direction perpendicular to the plane of incidence (the plane containing both the incoming and the reflected light of a light ray). R-parallel is correspondingly the parallel component. The reflectance is in general a strongly non-linear function of the incidence angle. (b) For the angles of relevance for this work (less than $15^{\circ}$ ) the reflectance may be approximated by the value at zero incidence angle. The reflectance is dependent on the refractive index of the liquid sample. For a refractive index, $n_{t}$, of 1.38 (e.g. $25 \%$ sodium chloride solution) the reflectance is approximately $25 \%$ higher than for a refractive index of 1.33 (e.g. water). c) Difference data between images of $25 \%$ and $0 \%$ sodium chloride solutions. The specular part of Fig. 1b (the high intensity area) is here showing a positive reflectance difference due to the increased refractive index (note the rotation of the figure compared to Fig. 1b). 
a)

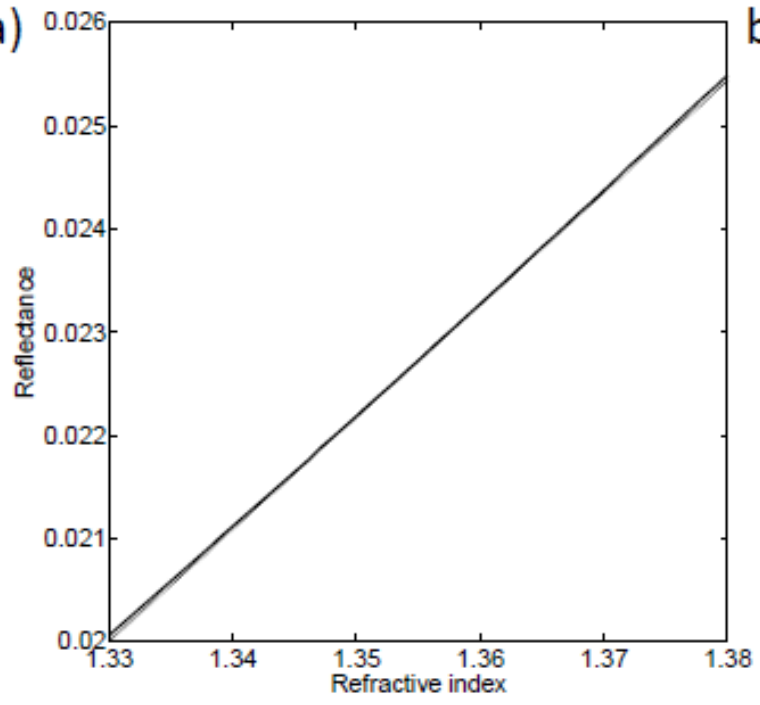

b)

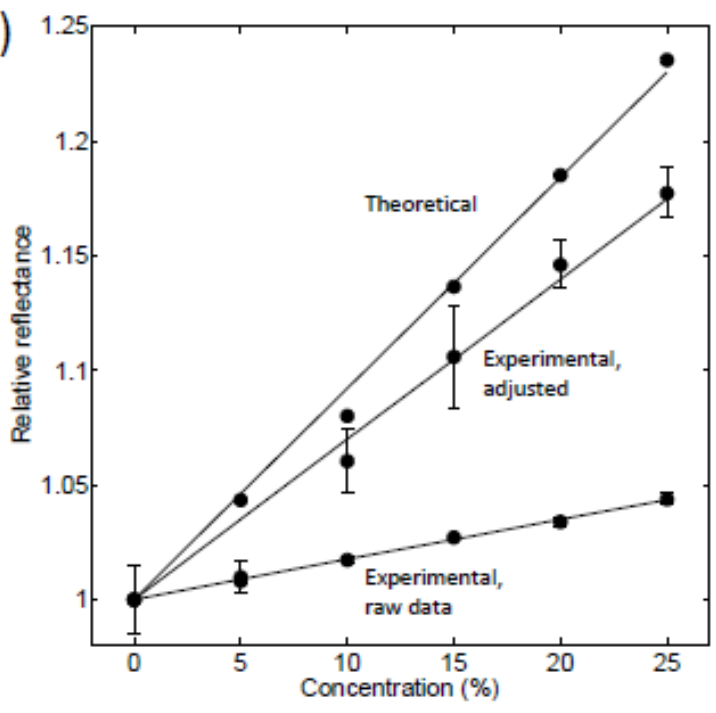

Fig. 3. (a) Reflectance, $R$, at normal incidence according to eq. (1) for refractive indices in the range $1.33<n_{t}<1.38$. In this region $R$ is approximately linearly dependent on $n_{t}$ as can be seen by comparison with the straight line (dotted). (b) Theoretical and experimental relative reflectance, i.e. the reflectance at a certain concentration divided by the (mean) reflectance at zero concentration, for different weight concentrations of sodium chloride in de-ionized water. Mean values and standard deviations for 5 measurements are shown for the experimental data as well as least squares linear curve fits. The standard deviation of the raw data points are smaller than the data points at this scale, but can be studied at a different scale in Fig. 4. 


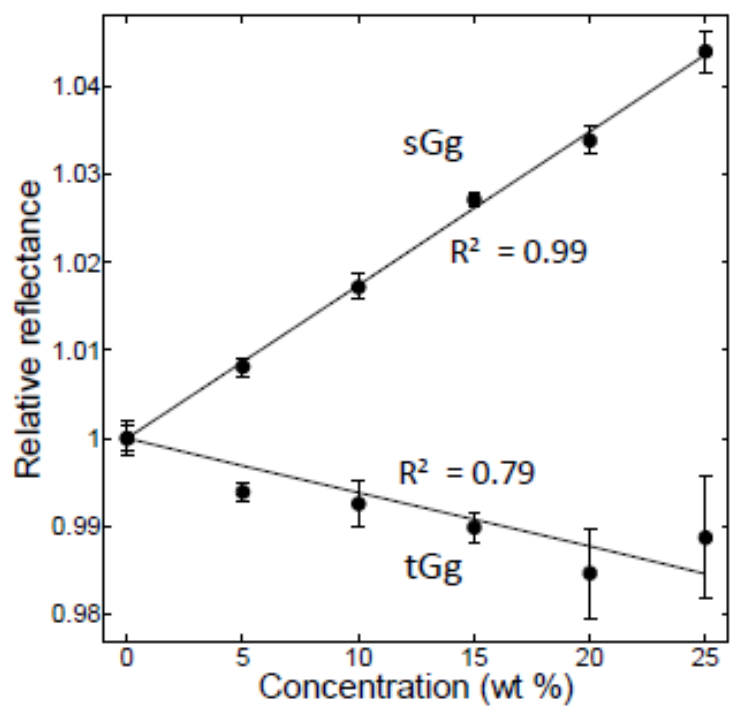

Fig. 4. Gg (green illumination and green detection channel) relative reflectance as a function of sodium chloride concentration. sGg is data from Area 2 (see Fig. 1b) including specular reflected light from the air-liquid interface and $\mathrm{tGg}$ is data from Area 1 dominated by transmitted light diffusively reflected at the liquid-vessel interface. Mean values and standard deviations for 5 measurements are shown as well as least squares linear curve fits. The $\mathrm{R}^{2}$ value is the coefficient of determination, which is a measure of the goodness of the curve fit. 
a)

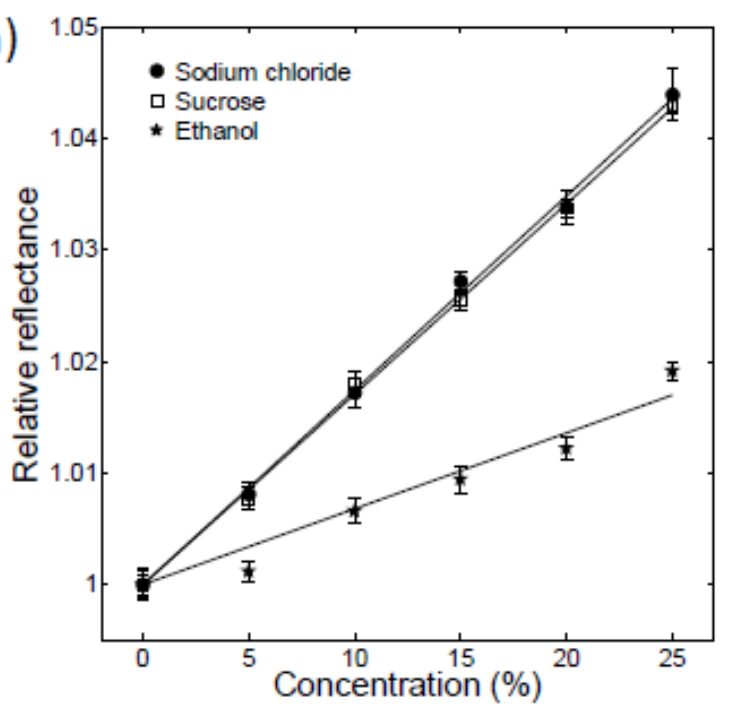

b)

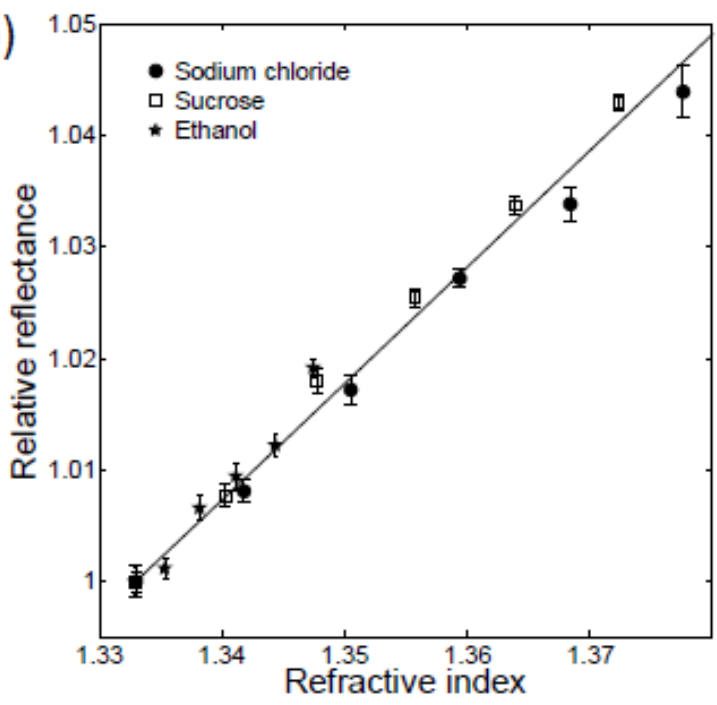

Fig. 5. (a) Relative reflection for solutions with different concentrations of sodium chloride, sucrose and ethanol. For the first two substances the concentrations are in wt $\%$ and for ethanol in vol $\%$. Mean values and standard deviations for 5 measurements, measured in randomized order, are shown as well as least squares linear curve fits. The measurements were repeated once a day during 5 days. The slope of the fitted lines increase in order of increasing refractive index of the solutions. (b) The same data with concentration data converted to refractive index via tabulated data [17]. 

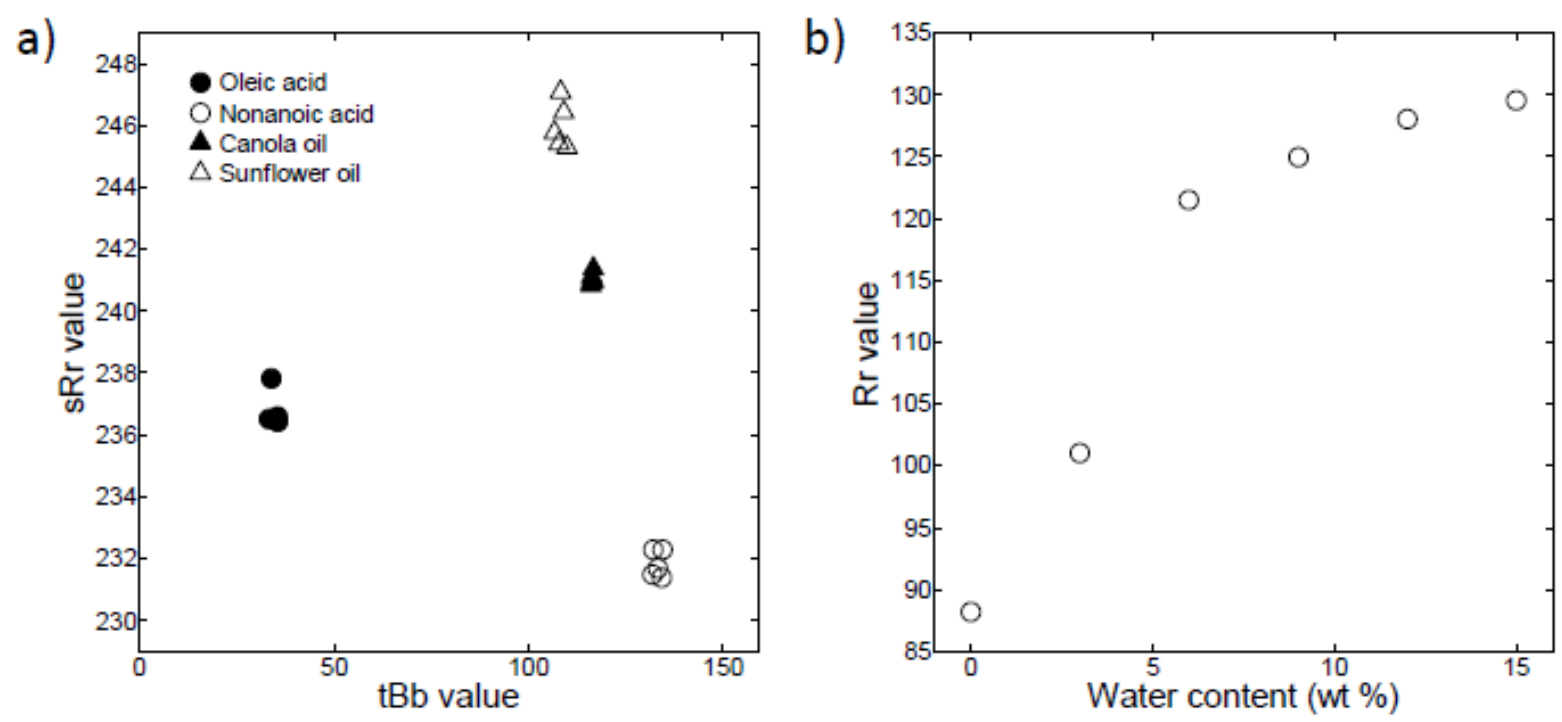

Fig. 6. (a) Classification of edible oils (canola oil and sunflower oil) and pure fatty acids (oleic acid and nonanoic acid). Blue illumination detected in the blue channel (Bb) from Area 1 is the $\mathrm{tBb}$ parameter and red illumination in the red channel (Rr) from Area 2 is the sRr parameter. The tBb and sRr values are image intensities in the range 0-255 (8 bit resolution) in the blue (b) and red (r) channels, respectively. The measurements were repeated once a day during 5 days. (b) Influence of water content in the canola oil, after shaking to obtain an emulsion, on the $\mathrm{Rr}$ value (red illumination in the red channel measured in Area 1). 
a)

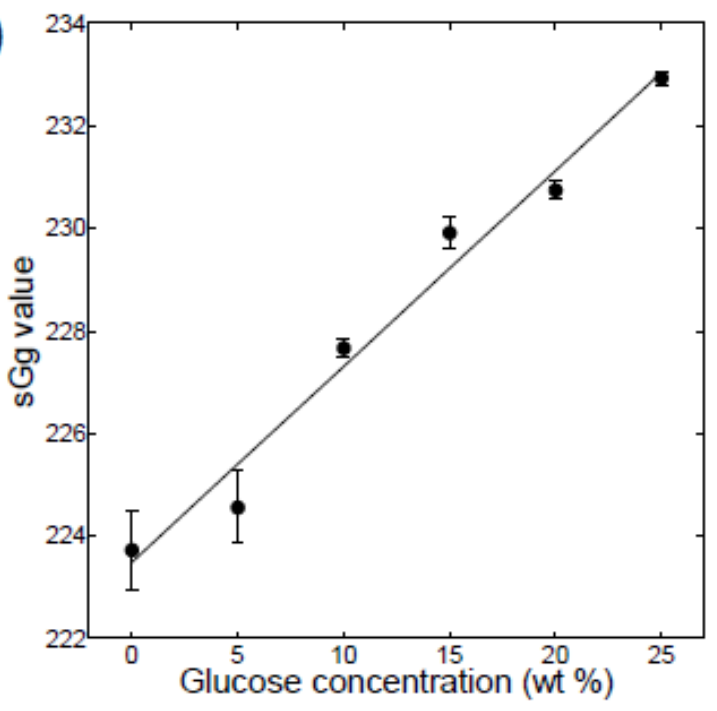

b)

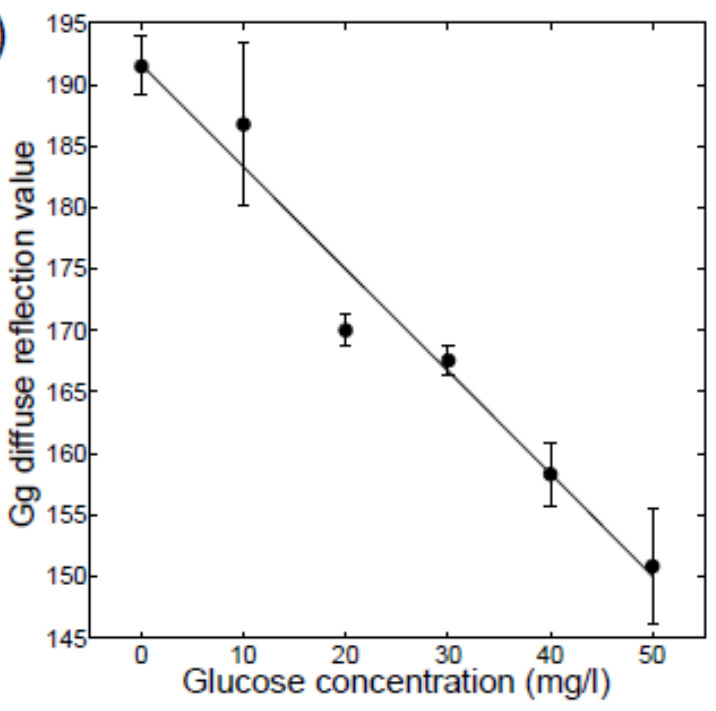

Fig. 7. Glucose measurements in two concentration regions. In (a) high concentrations are investigated by direct sGg measurements in the solution. In (b) low concentrations are detected with $\mathrm{Gg}$ diffuse reflection by direct imaging of glucose analyzing test strips. Mean values and standard deviations for (a) 5 measurements during 5 days and (b) 3 measurements during 3 days are shown, respectively. The measured values are image intensities in the range 0-255 in the green $(\mathrm{g})$ channel of the camera. 


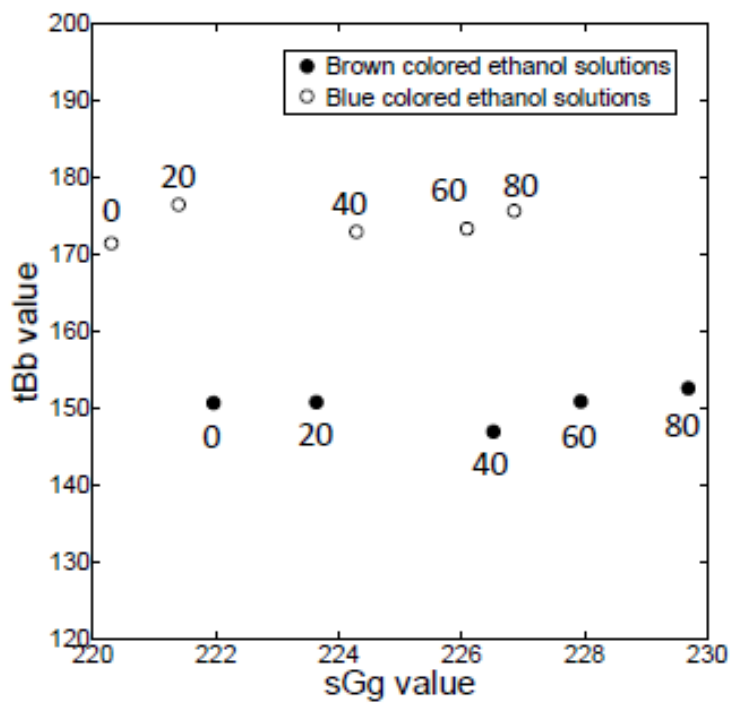

Fig. 8. A combination of $\mathrm{sGg}$ and $\mathrm{tBb}$ measurements enables classification of different ethanol concentrations (numbers are in vol \% in the figure) in two differently colored solutions. The measured values are image intensities in the range $0-255$ in the green $(\mathrm{g})$ and blue (b) channels, respectively. 